\title{
Autoimmune thyroiditis: Association with two common polymorphisms of the RAGE gene and oxidative stress
}

Giannakou M ${ }^{1}$, Saltiki K ${ }^{1,2}$, Mantzou E 2, Loukari E 2, Philippou G 2 ${ }^{2}$ Terzidis K 2, Lili K², Stavrianos C 2, Alevizaki M ${ }^{1,2}$

${ }^{1}$ Endocrine Unit, Dept Med Therapeutics, ${ }^{2}$ Dept of Endocrinology, Metabolism and Diabetes, Athens University School of Medicine, Greece

\section{Introduction - Aim of the study}

Polymorphisms of the receptor for advanced glycation end products (AGEs), RAGE, have been associated with autoimmune disorders and their complications. There are no studies concerning Hashimoto's thyroiditis; the aim of our study was to investigate the possible role of two RAGE polymorphisms (-429T>C, $-374 T>A)$ in combination with indices of oxidative status in women with Hashimoto's thyroiditis (HT).

\section{Patients and Methods}

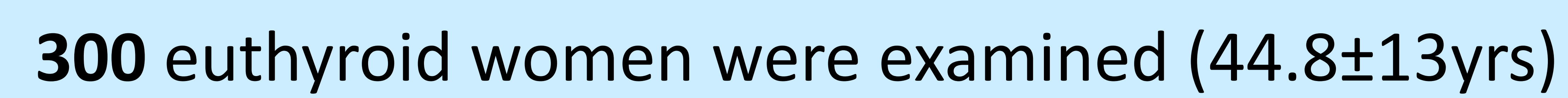
205 women had HT all with positive thyroid antibodies [ThAb (+)]:

\section{- 96 with treatment}

- 109 without treatment

95 women with $\operatorname{ThAb}(-)$ and negative family history for HT (control group)
For the evaluation of oxidative stress, total lipid peroxide levels in serum (TOS) were measured.

The RAGE polymorphisms -429T>C Alu I and -374T>A Mfe I were studied by RFLP in genomic DNA.

\section{Results}

- Women with HT on T4 had higher TOS levels compared to those without treatment and to controls (mean TOS: $520 \mu \mathrm{mol} / \mathrm{l}$ vs $421.04 \mu \mathrm{mol} / \mathrm{l}$ vs $447.6 \mu \mathrm{mol} / \mathrm{l}$, respectively, $p=0.026$, figure 1 ).

- The prevalence of -429T>C RAGE polymorphism was significantly higher in this group compared to HT without treatment and controls ( $18.8 \%$ vs $11.9 \%$ vs $6.3 \%$ respectively, $p=0.032$, figure 2 ).

- In the entire cohort, increased TOS and carrying the $-429 \mathrm{~T}>\mathrm{C}$ polymorphism were independent predictors of HT (OR1.64, OR1.60 respectively, figure 3). The coexistence of these factors had an additive effect (OR 5.4, figure 3 ).

- There was no difference in the prevalence of -374T>A polymorphism between the studied groups.

Figure 1

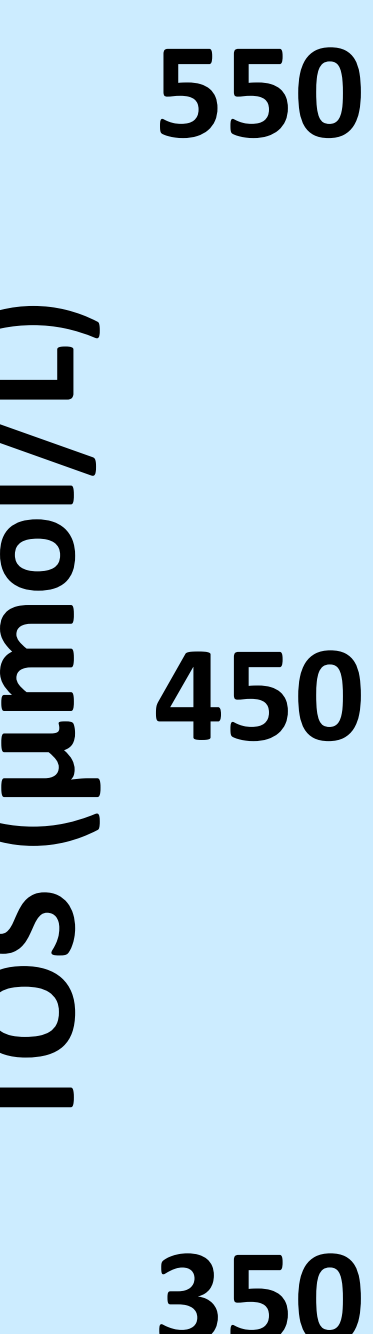

350

HT with HT treatment without Control treatment
Figure 2

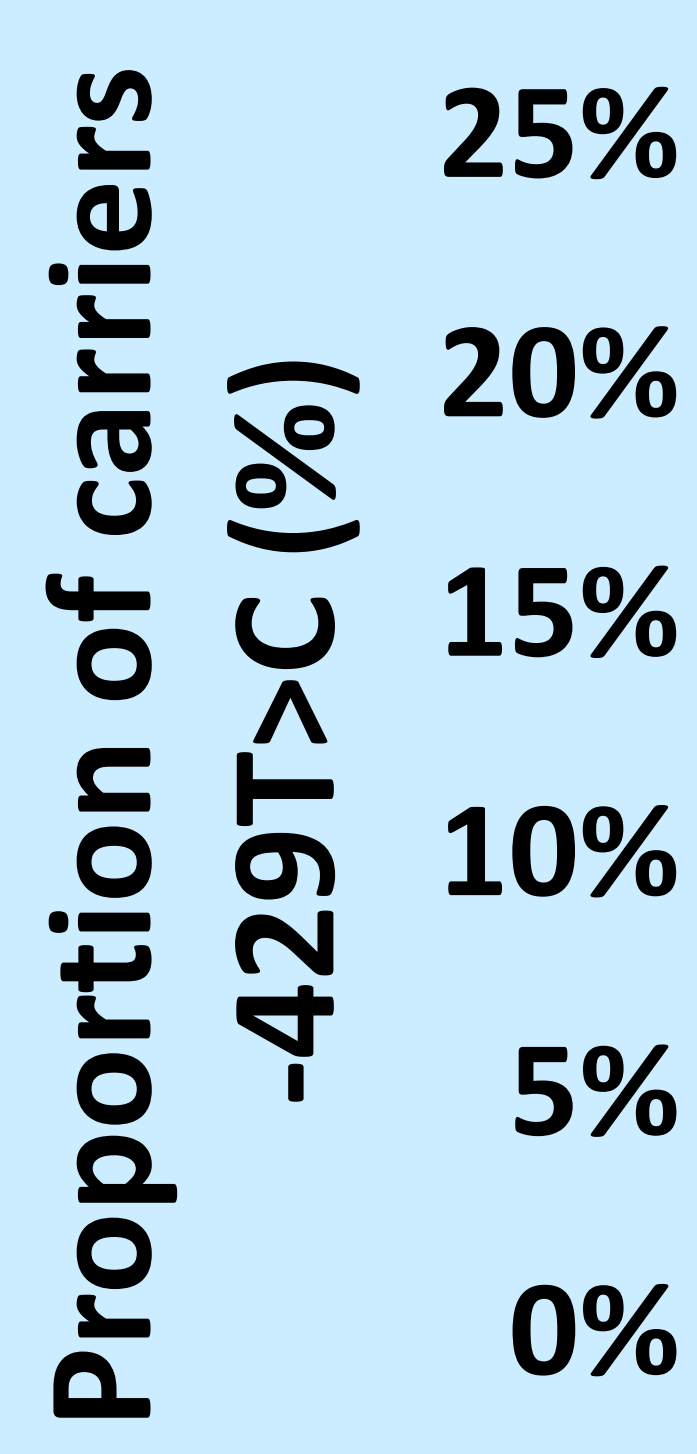

$0 \%$

HT with HT without treatment treatment

\section{Conclusions}

Figure 3
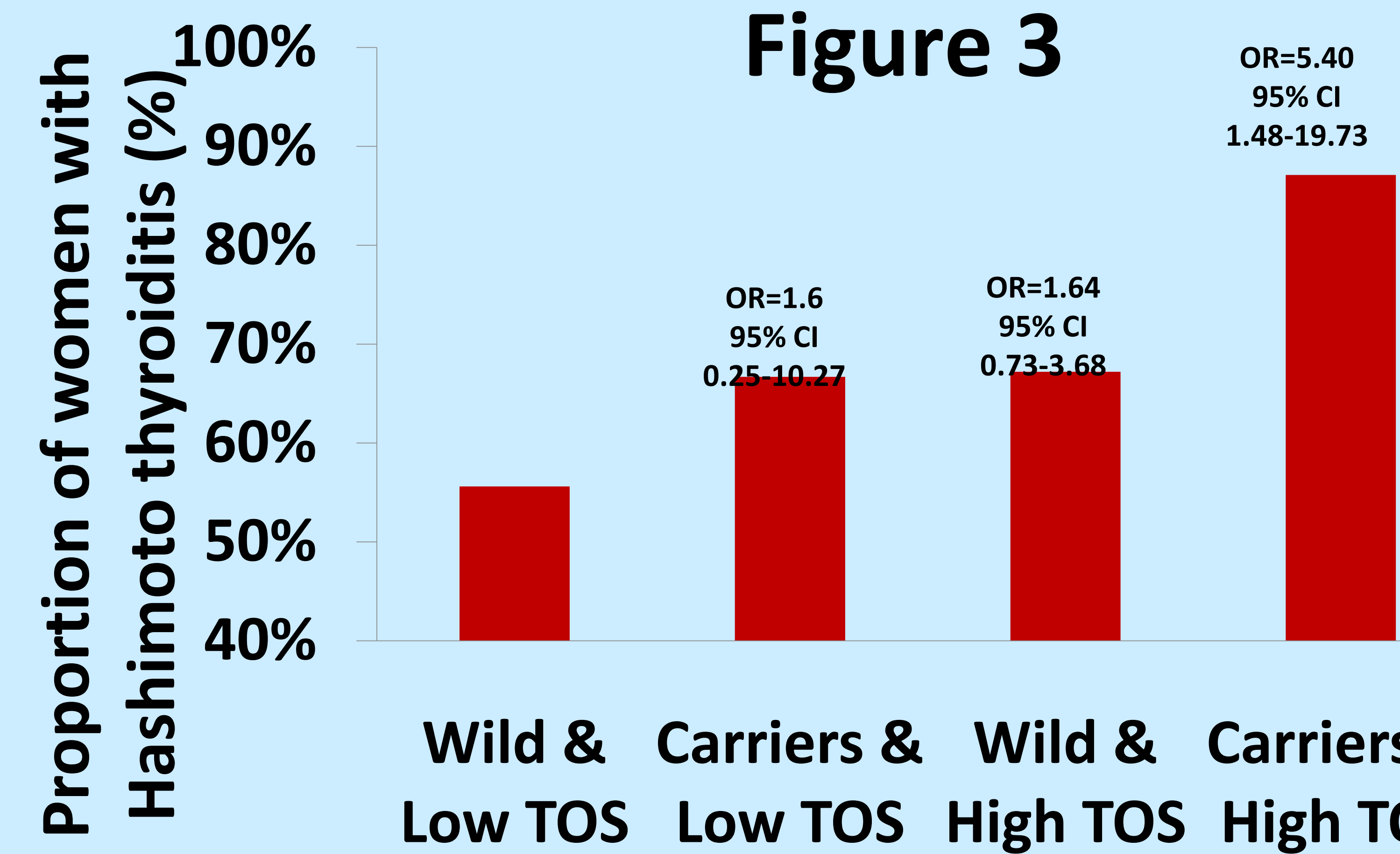

Wild \& Carriers \& Wild \& Carriers \& Low TOS Low TOS High TOS High TOS

Women with increased TOS levels who are also carriers of the -429T>C polymorphism of RAGE are at increased risk to have Hashimoto's thyroiditis and receive T4 replacement. These findings possibly suggest a role of this system in the elevated oxidative stress accompanying autoimmune thyroiditis. 